\title{
Vorbemerkungen zu diesem Buch: Quellen und Zitierweise
}

Hinweise zur Zitation: Kants Schriften und Äußerungen werden im Allgemeinen nach der Akademie-Ausgabe (Berlin $1900 \mathrm{ff}$.) zitiert, und zwar nach dem folgenden Muster: Siglum, AA Band-Nummer: Seite.Zeile. Also zum Beispiel: GMS, AA 04:389.27. Die Siglen folgen dem Siglenverzeichnis für Publikationen in den KantStudien. Reflexionen sind zusätzlich mit „R“ sowie ihrer Nummer nach der Akademie-Ausgabe gekennzeichnet. Also zum Beispiel: R 5237.

Ausnahmen bilden Zitate aus der Vorlesungsnachschrift von Kaehler, die nicht in der Akademie-Ausgabe enthalten ist. Hier wird nach der folgenden Ausgabe zitiert: Immanuel Kant (2004): Vorlesungen zur Moralphilosophie herausgegeben von Werner Stark sowie ein Zitat aus: Immanuel Kant's Menschenkunde oder philosophische Anthropologie (Nach den handschriftlichen Vorlesungen) 1831 herausgegeben von Friedrich Starke. Außerdem wird für mündliche Äußerungen Kants auf die folgende Sammlung zurückgegriffen: Immanuel Kant in Rede und Gespräch (1990) herausgegeben von Rudolf Malter.

Alle anderen Zitate werden nach dem amerikanischen Verfahren, also durch Angabe von Autor, Erscheinungsjahr und Seitenzahl, gekennzeichnet. Also zum Beispiel: Ludwig (2013), S. 59. Die weiteren bibliographischen Angaben sind dem Literaturverzeichnis zu entnehmen.

Kants Hervorhebungen sind generell durch Sperrschrift gekennzeichnet. Andere und zusätzliche Hervorhebungen im Original - zum Beispiel durch Fettdruck - werden im Sinn einer diplomatischen Textwiedergabe ebenfalls übernommen, so dass es in wenigen Einzelfällen zu doppelten Hervorhebungen kommen kann. Im Hinblick auf Orthographie, Grammatik und Interpunktion ist die Wiedergabe ebenfalls diplomatisch, d.h. sämtliche Eigenheiten und auch offensichtliche Fehler werden übernommen. Alle eingerückten Kant-Zitate werden mit einem Punkt als Satzschlusszeichen abgeschlossen, auch wenn dies im Original nicht der Fall ist. Eigene Hervorhebungen werden kursiviert und mit einem entsprechenden Hinweis [Hervorhebung UR] versehen. Fremdsprachliche, v. a. lateinische Ausdrücke in den Kant-Zitaten werden ebenfalls kursiv geschrieben.

Darüber hinaus erscheinen Titel, Überschriften und Teilüberschriften ebenfalls kursiv. Wenn also beispielsweise von dem Werk Tugendlehre - als abkürzende Bezeichnung für den eigentlichen Titel: Metaphysische Anfangsgründe der Tugendlehre - die Rede ist, erscheint der Titel kursiv; ist allerdings nicht vom Werk, sondern z. B. von der Tugendlehre als inhaltlichem Lehrstück die Rede, dann wird 
der Begriff nicht kursiviert. Vorlesungsnachschriften werden durch Kursiv-Druck des jeweiligen Namensgebers akzentuiert, also z. B. Vigilantius-Nachschrift.

Quellenangaben erfolgen in der Regel unmittelbar nach einem Zitat oder nach Sinneinheiten; stammen allerdings mehrere aufeinanderfolgende Zitate aus derselben Quelle und derselben Seite, erfolgt nur ein Quellennachweis nach dem letzten Zitat.

Hinweise auf Abschnitte (z. B. Abschnitt 3.2) beziehen sich als Querverweise stets auf Abschnitte in diesem Buch. 